\section{Arbres oléagineux locaux sous-utilisés : potentialités pour une promotion des chaînes de valeur des huiles et implications pour la gestion durable des ressources dans le Kénédougou, Ouest du Burkina Faso}

Fanta Reine Sheirita TIÉtıAmbou

\section{RÉSUMÉ}

Au Burkina Faso, il existe des arbres oléagineux locaux à fort potentiel mais sous-utilisées dont la promotion peut contribuer à générer des revenus substantiels pour les communautés rurales et urbaines. La présente étude vise à évaluer le potentiel des Chaînes de Valeur (CVA) des huiles d'arbres locaux pour l'amélioration de l'économie rurale et la gestion durable des ressources dans la province du Kénédougou (Ouest du Burkina Faso). Des enquêtes ethnobotaniques, un bilan de masse de la production des huiles et une étude de marché des huiles et produits oléagineux ont été conduits. Pour comprendre la disponibilité des ressources, une estimation de la production fruitière des arbres ainsi qu'une évaluation de la structure des peuplements ont été réalisées.

Les résultats révèlent 11 plantes oléagineuses connues parmi lesquelles Carapa procera DC., Lophira lanceolata Van Tiegh. ex Keay et Pentadesma butyracea Sabine ont un grand potentiel de valorisation. Le procédé d'extraction traditionnelle de l'huile de C. procera est typiquement artisanal avec une efficacité permettant d'obtenir $65,49 \%$ de la teneur en huile de la graine. Pour $L$. lanceolata et $P$. butyracea, l'extraction de l'huile est semi artisanale avec des efficacités respectives de $69,08 \%$ et $75,21 \%$ de la teneur en huile des graines. Les indices d'acidité et de paraanisidine des huiles extraites restent dans les limites de valeurs acceptables du Codex Alimentarius. Dans la filière des huiles de ces trois arbres oléagineux, sept CVA ont été identifiées dont trois sont incitatives pour la promotion. Ce sont le beurre brut de $P$. butyracea destiné à l'exportation avec $88,23 \%$ de Chance de Contribution à la Croissance pro Pauvre du Burkina Faso (CCCP-BF), le savon médical artisanal de $C$. procera $(79,41 \%$ de CCCP-BF) et le savon médical industriel de $C$. procera (73,5\% de CCCP-BF). L'analyse de la CVA du savon médical artisanal montre qu'elle contribue à une croissance enrichissante globale d'un taux de $77,76 \%$. Soixante-dix pour-cent des opérateurs ont moins de $20 \%$ du revenu total de la CVA qui est actuellement de 22 904,2 Euros/an. Ce revenu total augmenterait à au moins 51929,1 Euros/an si les opérateurs s'engageaient dans la promotion de la CVA.

Mots-clés : arbres oléagineux, chaînes de valeurs ajoutées, communautés rurales, huiles locales, produits forestiers non ligneux, savoirs locaux.

\section{Local under-used oil-seed tree species: potential fo promoting oil-seed value chains and implications for the sustainability of managed resources in the Kénédougou province in western Burkina Faso}

\section{ABSTRACT}

Burkina Faso's forests have high but under-used potential for local oil-seed production, which deserves to be promoted as a substantial source of income for rural and urban communities. This study aimed to assess the potential for establishing added-value chains based on local oil-seed trees to enhance the local economy and the sustainability of managed resources in the Kénédougou province in western Burkina Faso. To do so, we conducted ethnobotanical surveys, a mass-balance assessment of oil production and a market survey on oils and oil products. To better understand resource availability, an estimation of oil-seed production and the structure of stands were assessed. The results showed that 11 well-known oleaginous species, including Carapa procera DC., Lophira lanceolata Van Tiegh. ex Keay and Pentadesma butyracea Sabine, have high oil production potential. The traditional extraction process for $C$. procera oil is characteristically artisanal, yielding $65.49 \%$ of the total oil content of the seeds. For L. lanceolata and P. butyracea, oil extraction is semi-artisanal, yielding $69.08 \%$ and $75.21 \%$ respec tively of the total oil content. The acidity and para-ani sidine indices of extracted oils were ranged within acceptable values of the Codex Alimentarius. Seven potential value-added chains were identified for products from these three oil-seed species, three of which deserve to be promoted: the unprocessed $P$. butyracea butter for export, with an $88.23 \%$ Chance of Contributing to Poverty-reducing Growth in Burkina Faso (CCCP-BF), the artisanal medicinal soap of $C$. procera $(79.41 \%)$ and industrially produced medicinal soap of C. procera $(73.5 \%)$. Analysis of the value-added chain for artisanal medicinal soap shows a $77.76 \%$ chance of contribution to overall wealth-producing growth. Seventy percent of operators earn less than $20 \%$ of the total turnover from the value-added chain, currently 22,904.2 Euros/year. This total income would increase to at least 51,929.1 Euros/year if the operators were to engage in promoting the value-added chain.

Keywords: oil-seed trees, added-value chains, rural communities, local oils, non-timber forest products, local knowledge.

\section{Árboles oleaginosos locales infrautilizados: posibilidades de desarrollo de las cadenas de valor agregado de los aceites e implicaciones para el manejo sostenible de los recursos en Kénédougou, Burkina Faso occidental}

\section{RESUMEN}

En Burkina Faso existen árboles oleaginosos con gran potencial, pero que están infrautilizados. Su desarro llo puede contribuir a generar considerables ingresos para las comunidades rurales y urbanas. Este estudio pretende evaluar el potencial de las Cadenas de Valor Agregado (CVA) de aceites de árboles locales para mejorar la economía rural y el manejo sostenible de los recursos en la provincia de Kénédougou (Burkina Faso occidental). Se llevaron a cabo encuestas etno botánicas, un balance de masa de la producción de aceites y un estudio de mercado de aceites y productos oleaginosos. Para comprender la disponibilidad de los recursos, se realizaron una estimación de la produc ción frutal de los árboles y una evaluación de la estructura de los rodales.

Los resultados muestran 11 plantas oleaginosas cono cidas entre las que destacan, por su gran potencial de valorización, Carapa procera DC., Lophira lanceolata Van Tiegh. ex Keay y Pentadesma butyracea Sabine. El proceso de extracción tradicional del aceite de $C$. procera es totalmente artesanal con una eficacia que permite extraer el $65,49 \%$ del contenido de aceite de la semilla. En L. lanceolata y P. butyracea, la extracción de aceite es semiartesanal con eficacias del $69,08 \%$ y $75,21 \%$, respectivamente, del contenido de aceite de la semilla. Los índices de acidez y para-Anisidina de los aceites extraidos se sitúan en unos valores límites aceptables del Codex Alimentarius. En el circuito de los aceites de estos tres árboles oleaginosos, se identificaron siete CVA, tres de la cuales presentan alicientes para su desarrollo. Se trata de la manteca bruta de $P$. butyracea destinada a la exportación, con un $88,23 \%$ de Posibilidad de Contribución al Crecimiento Pro-pobre de Burkina Faso (PCCP-BF), el jabón medicinal artesano de $C$. procera $(79,41 \%$ de PCCP-BF) y el jabón medicinal industrial de $C$. procera $(73,5 \%$ de PCCP-BF). El análisis de la CVA del jabón medicinal artesano muestra que la cadena contribuye a un crecimiento enriquecedor global con una tasa del $77,76 \%$. El setenta por ciento de los actores del sector recibe menos del $20 \%$ de los ingresos totales de la CVA, que actualmente es de 22 904,2 euros/año. Dichos ingresos totales aumentarían al menos hasta 51 929,1 euros/año si los actores se comprometieran en el desarrollo de la CVA.

Palabras clave: árboles oleaginosos, cadenas de valor agregado, comunidades rurales, aceites locales, productos forestales no madereros, saberes locales.

\section{Grade et diplôme : Docteur}

Université : Université Ouaga I Pr Joseph KI-ZERBO,

Burkina Faso.

Date de soutenance : 14 novembre 2017

Composition du jury

Direction : Amadé OUedRaogo (Maître de Conférences, Université Ouaga I Professeur Joseph Ki-Zerbo, Laboratoire de Biologie et Écologie Végétales, Burkina Faso)

Membres : Issaka Joseph Boussım (Professeur Titulaire, Université Ouaga I Professeur Joseph Ki-Zerbo, Laboratoire de Biologie et Écologie Végétales, Burkina Faso), Romain GLĖLÉ KAKAï (Professeur Titulaire, Université d'Abomey-Calavi, Laboratoire de Biomathématiques et d'Estimation Forestières, Bénin), Abdelkader AïT EL MEKK (Professeur Titulaire, École Nationale d'Agriculture de Meknès, Département d'Économie Rurale, Maroc), Adjima Thıombiano (Professeur Titulaire, Université Ouaga I Professeur Joseph Ki-Zerbo, Laboratoire de Biologie et Écologie Végétales, Burkina Faso), Sita GuINKo (Professeur Titulaire, Université Ouaga I Professeur Joseph Ki-Zerbo, Laboratoire de Biologie et Écologie Végétales, Burkina Faso).

\section{Langue de rédaction : Français}

Accès au manuscrit :

\section{Contact :}

Adresse postale : Université Ouaga I Joseph Ki-Zerbo, UFR en Sciences de la Vie et de la Terre, Laboratoire de Biologie et Écologie Végétales, 03 BP 7021, Ouagadougou 03, Burkina Faso. Tél. : 0022673143444 tietiambou.fanta@gmail.com

\section{Publications / Publications / Publicaciones}

Bazongo P., Tiétiambou F. R. S., Diallo D. A. Kouyaté A. M., Lykke A. M., Ouédraogo A., Bassolé I. H. N., 2016. Production locale améliorée de l'huile de Carapa procera. Fiche technique. Projet QualiTree, 2 p. http://qualitree.neri.dk/index. php?page=technical-leaflet-of-carapa-procera-oil Tiétiambou F. R. S., Lykke A.M., Korbéogo G., Thiombiano A., Ouédraogo A., 2016. Perceptions et savoirs endogènes sur les espèces oléagineuses locales dans le Kénédougou (Burkina Faso). Bois et Forêts des Tropiques, 327 : 39-50. https://doi.org/10.19182/ bft2016.327.a31295

Tiétiambou F. R. S., Bazongo P., Diallo D. A., Kouyaté A. M., Lykke A. M., Bassolé I. H. N., Ouédraogo A., 2015. Production locale améliorée de l’huile de Balanites aegyptiaca. Fiche technique. Projet QualiTree, 2 p. http://qualitree.neri.dk/index.php?page=technical-leaflet-of-bananites-aegyptiaca-oil

Tiétiambou F. R. S., Bazongo P., Diallo D. A., Kouyaté A. M., Lykke A. M., Bassolé I. H. N., Ouédraogo A. 2016. Production locale améliorée de l'huile de Pentadesma butyracea. Fiche technique. Projet QualiTree, 2 p. http://qualitree.neri.dk/index.php?page=technical-leaflet-of-pentadesma-butyracea-oil 
Bois et Forêts des Tropiques - ISSN: L-0006-579X

Volume 335 - $1^{\text {st }}$ quarter - January 2018 - p. 71-72

Planche photographique.

Différents produits des trois arbres olégineux à fort potentiel de valorisation : fruits $(1 \mathrm{a}-\mathrm{c})$, graines $(2 \mathrm{a}-\mathrm{c})$ et huile (3a-c), respectivement de Carapa procera (a), Lophira lanceolata (b) et Pentadesma butyracea (c).

La planche photographique illustre la matière première (fruits et graines) des trois espèces (C. procera, $L$. lanceolata et $P$. butyracea) dans leur habitat naturel et leur transformation en huile. Les fruits et les graines sont ramassés dans la forêt par les femmes. La matière première est transformée par des procédés traditionnels pour en extraire l'huile. L'huile obtenue est directement consommée, vendue ou utilisée pour la fabrication d'autres produits tels que le savon.

Photos (1a, 2a et 3a), B. Lankoandé ; Photos (2a, 2b, 2c, 3a, 3b et 3c), F. Tiétiambou.

Products from the three oil-seed trees with high value-added potential: fruits (1a-c), seeds (2a-c) and oil (3a-c), respectively from Carapa procera (a), Lophira lanceolata (b) and Pentadesma butyracea (c).

The photographic plate shows the raw material (fruits and seeds) of the three species (C. procera, L. lanceolata and P. butyracea) in their natural habitat and extraction of their oil. The fruits and seeds are gathered in the forest by women. The raw material is then processed by artisanal methods to extract the oil, which is then consumed directly, sold or used to manufacture other products such as soap.

Photos (1a, 2a et 3a), B. Lankoandé ; Photos (2a, 2b, 2c, 3a, 3b et 3c), F. Tiétiambou.

Distintos productos de tres árboles oleaginosos con alto potencial de valorización: frutos $(1 \mathrm{a}-\mathrm{c})$, semillas $(2 \mathrm{a}-\mathrm{c})$ y aceite $(3 \mathrm{a}-\mathrm{c})$ de Carapa procera (a), Lophira lanceolata (b) y Pentadesma butyracea (c), respectivamente.

Las reproducciones fotográficas muestran la materia prima (frutos y semillas) de las tres especies (C. procera, L. lanceolata y $P$. butyracea) en su hábitat natural y su transformación en aceite. Las mujeres recolectan los frutos y semillas en el bosque. Esta materia prima se transforma mediante procesos tradicionales para extraer el aceite. Dicho aceite se consume directamente, se vende o se emplea para fabricar otros productos como el jabón.

Fotos (1a, 2a y 3a), B. Lankoandé; Fotos (2a, 2b, 2c, 3a, 3b y 3c), F. Tiétiambou.

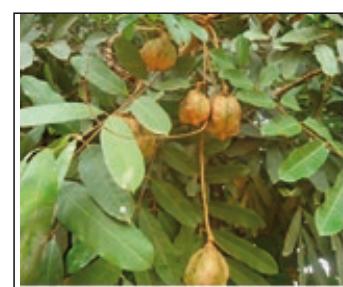

1 (a)

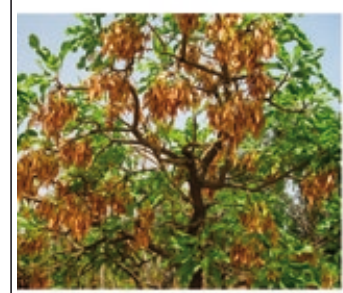

1 (b)

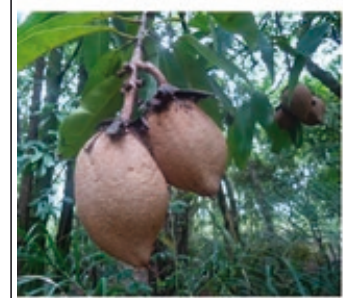

1 (c)

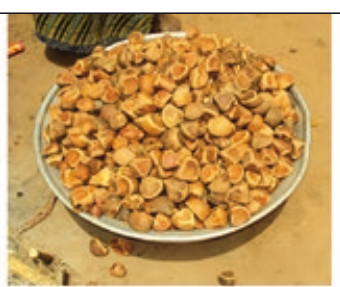

2 (a)

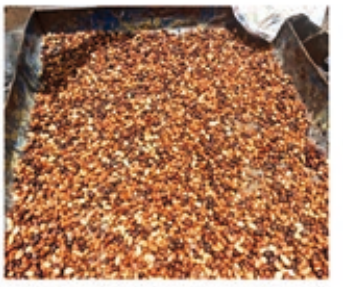

2 (b)

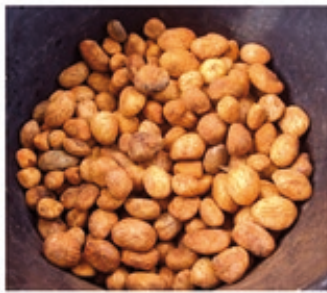

2 (c)

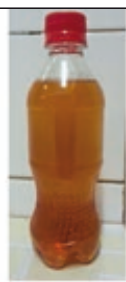

3 (a)

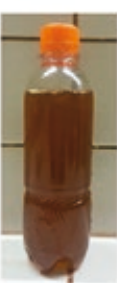

3 (b)

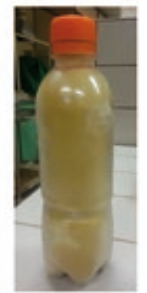

3 (c)

Figure 1.

Ensemble des acteurs intervenants dans la chaîne de valeur du Savon Médical Artisanal à base de l'huile des graines de Carapa procera au Burkina Faso.

La figure illustre les acteurs qui interviennent dans l'exploitation des ressources de l'espèce $C$. procera pour le développement de la Chaîne de valeur du Savon Médical Artisanal à base d'huile de cette espèce (SMACp). Ces acteurs se répartissent en sept catégories aux niveaux micro-économique et méso-économique. Les communautés rurales à la base occupent les principales fonctions micro-économiques de collecte de la matière première, d'extraction de l'huile brute et de la production de savons. L'ensemble des acteurs de ces catégories est relié à travers 22 liens. Ces relations concernent pour la plupart des accords qui sont plus forts au niveau des maillons de la commercialisation et de la transformation. Parallèlement, il y a quelques acteurs qui opèrent individuellement et écoulent leurs productions à des groupements à travers des échanges aléatoires de marché.

Flow diagram showing all players involved in the value chain for artisanal medicinal soap based on oil from Carapa procera seeds in Burkina Faso.

The diagram shows the players involved in using C. procera resources to develop the value chain for Artisanal Medicinal Soap produced from the oil of this species (AMSCp). These players fall into seven micro- to middle economic categories. The primary functions are carried out by the rural communities who gather the raw materials, extract the raw oil and produce the soap. Altogether, 22 interconnections link the different players in the chain. These points of interconnection are mainly agreements that are more binding between the processing and marketing stages. In parallel, some players operate individually, selling their products to marketing groups as and when market opportunities arise.

Conjunto de actores que intervienen en la cadena de valor del jabón medicinal artesanal a base de aceite de semillas de Carapa procera en Burkina Faso.

La figura muestra a los actores que intervienen en el aprovechamiento de los recursos de $C$. procera para el desarrollo de la cadena de valor del jabón medicinal artesano a base del aceite de esta especie (JMACp). Estos actores se distribuyen en siete categorías en niveles microeconómico y mesoeconómico. Las comunidades rurales ocupan fundamentalmente las principales funciones microeconómicas: recolección de la materia prima, extracción del aceite bruto y producción de jabones. Todos los actores de estas categorías se interrelacionan a través de 22 vínculos. Estas relaciones conciernen sobre todo acuerdos, que son más sólidos en los eslabones de la comercialización y transformación. Paralelamente, hay algunos actores que operan individualmente y venden su producción a grupos económicos mediante intercambios aleatorios de mercado.

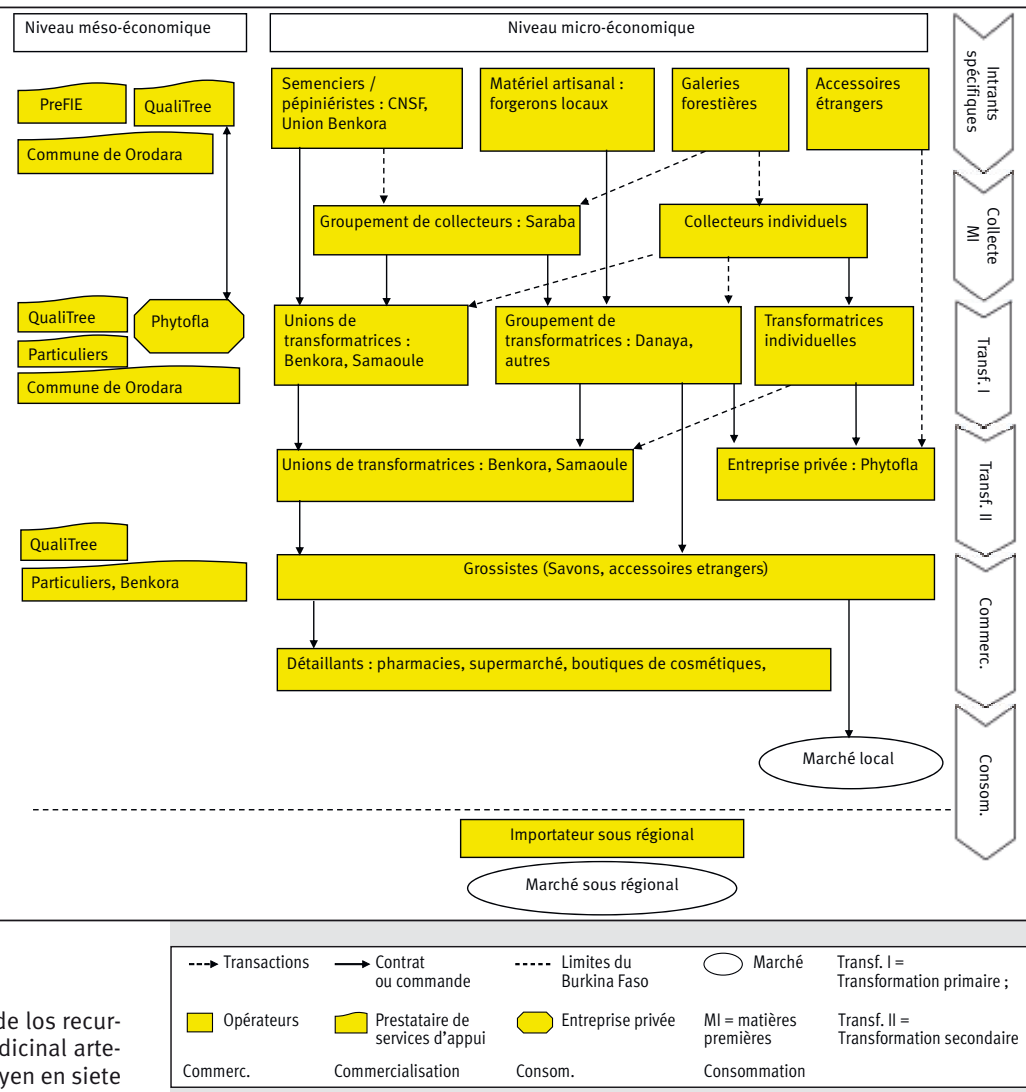

Légende :

CNSF : Centre National des Semences Forestières ; PreFIE : Fonds d'Investissement pour l'Environnement du programme d'appui au secteur forestier au Burina Faso ; QualiTree : Projet de recherche-développement basé sur les arbres oléagineux locaux en Afrique de l'Ouest. 